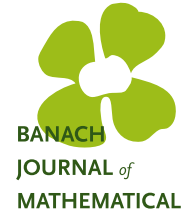

MATHEMATICAL

ANALYSIS

\title{
SCHATTEN-CLASS GENERALIZED VOLTERRA COMPANION INTEGRAL OPERATORS
}

\author{
TESFA MENGESTIE
}

Communicated by R. E. Curto

\begin{abstract}
We study the Schatten-class membership of generalized Volterra companion integral operators on the standard Fock spaces $\mathcal{F}_{\alpha}^{2}$. The Schatten $\mathcal{S}_{p}\left(\mathcal{F}_{\alpha}^{2}\right)$ membership of the operators are characterized in terms of $L^{p / 2}$-integrability of certain generalized Berezin-type integral transforms on the complex plane. We also give a more simplified and easy-to-apply description in terms of $L^{p}$-integrability of the symbols inducing the operators against super-exponentially decreasing weights. Asymptotic estimates for the $\mathcal{S}_{p}\left(\mathcal{F}_{\alpha}^{2}\right)$ norms of the operators have also been provided.
\end{abstract}

\section{INTRODUCTION AND MAIN RESULTS}

For functions $f$ and $g$, we consider the Volterra-type integral operator $V_{g}$ and its companion $I_{g}$ defined by

$$
V_{g} f(z)=\int_{0}^{z} f(w) g^{\prime}(w) d w \quad \text { and } \quad I_{g} f(z)=\int_{0}^{z} f^{\prime}(w) g(w) d w .
$$

Performing integration by parts in any one of the above integrals gives the relation

$$
V_{g} f+I_{g} f=M_{g} f-f(0) g(0)
$$

where $M_{g} f=g f$ is the multiplication operator induced by $g$. These integral operators have been studied extensively on various spaces of holomorphic functions with the aim to explore the connection between their behaviors with the functiontheoretic properties of the symbols $g$, especially after the works of Pommerenke

Copyright 2016 by the Tusi Mathematical Research Group.

Received Apr. 11, 2015; Accepted Jun. 7, 2015.

2010 Mathematics Subject Classification. Primary 47B32; Secondary 46E22, 46E20, 47B33.

Keywords. Fock space, Schatten class, Berezin transform, Volterra operator, generalized Volterra companion operator. 


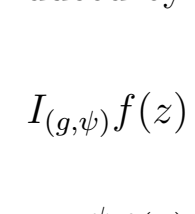

MATHEMATICAL

ANALYSIS

\title{
SCHATTEN-CLASS GENERALIZED VOLTERRA COMPANION INTEGRAL OPERATORS
}

\author{
TESFA MENGESTIE
}

Communicated by R. E. Curto

\begin{abstract}
We study the Schatten-class membership of generalized Volterra companion integral operators on the standard Fock spaces $\mathcal{F}_{\alpha}^{2}$. The Schatten $\mathcal{S}_{p}\left(\mathcal{F}_{\alpha}^{2}\right)$ membership of the operators are characterized in terms of $L^{p / 2}$-integrability of certain generalized Berezin-type integral transforms on the complex plane. We also give a more simplified and easy-to-apply description in terms of $L^{p}$-integrability of the symbols inducing the operators against super-exponentially decreasing weights. Asymptotic estimates for the $\mathcal{S}_{p}\left(\mathcal{F}_{\alpha}^{2}\right)$ norms of the operators have also been provided.
\end{abstract}

\section{INTRODUCTION AND MAIN RESULTS}

For functions $f$ and $g$, we consider the Volterra-type integral operator $V_{g}$ and its companion $I_{g}$ defined by

$$
V_{g} f(z)=\int_{0}^{z} f(w) g^{\prime}(w) d w \quad \text { and } \quad I_{g} f(z)=\int_{0}^{z} f^{\prime}(w) g(w) d w .
$$

Performing integration by parts in any one of the above integrals gives the relation

$$
V_{g} f+I_{g} f=M_{g} f-f(0) g(0)
$$

where $M_{g} f=g f$ is the multiplication operator induced by $g$. These integral operators have been studied extensively on various spaces of holomorphic functions with the aim to explore the connection between their behaviors with the functiontheoretic properties of the symbols $g$, especially after the works of Pommerenke

Copyright 2016 by the Tusi Mathematical Research Group.

Received Apr. 11, 2015; Accepted Jun. 7, 2015.

2010 Mathematics Subject Classification. Primary 47B32; Secondary 46E22, 46E20, 47B33.

Keywords. Fock space, Schatten class, Berezin transform, Volterra operator, generalized Volterra companion operator. 


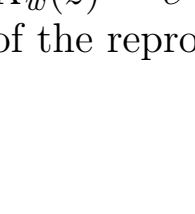

MATHEMATICAL

ANALYSIS

\title{
SCHATTEN-CLASS GENERALIZED VOLTERRA COMPANION INTEGRAL OPERATORS
}

\author{
TESFA MENGESTIE
}

Communicated by R. E. Curto

\begin{abstract}
We study the Schatten-class membership of generalized Volterra companion integral operators on the standard Fock spaces $\mathcal{F}_{\alpha}^{2}$. The Schatten $\mathcal{S}_{p}\left(\mathcal{F}_{\alpha}^{2}\right)$ membership of the operators are characterized in terms of $L^{p / 2}$-integrability of certain generalized Berezin-type integral transforms on the complex plane. We also give a more simplified and easy-to-apply description in terms of $L^{p}$-integrability of the symbols inducing the operators against super-exponentially decreasing weights. Asymptotic estimates for the $\mathcal{S}_{p}\left(\mathcal{F}_{\alpha}^{2}\right)$ norms of the operators have also been provided.
\end{abstract}

\section{INTRODUCTION AND MAIN RESULTS}

For functions $f$ and $g$, we consider the Volterra-type integral operator $V_{g}$ and its companion $I_{g}$ defined by

$$
V_{g} f(z)=\int_{0}^{z} f(w) g^{\prime}(w) d w \quad \text { and } \quad I_{g} f(z)=\int_{0}^{z} f^{\prime}(w) g(w) d w .
$$

Performing integration by parts in any one of the above integrals gives the relation

$$
V_{g} f+I_{g} f=M_{g} f-f(0) g(0)
$$

where $M_{g} f=g f$ is the multiplication operator induced by $g$. These integral operators have been studied extensively on various spaces of holomorphic functions with the aim to explore the connection between their behaviors with the functiontheoretic properties of the symbols $g$, especially after the works of Pommerenke

Copyright 2016 by the Tusi Mathematical Research Group.

Received Apr. 11, 2015; Accepted Jun. 7, 2015.

2010 Mathematics Subject Classification. Primary 47B32; Secondary 46E22, 46E20, 47B33.

Keywords. Fock space, Schatten class, Berezin transform, Volterra operator, generalized Volterra companion operator. 


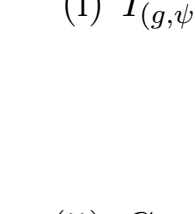

MATHEMATICAL

ANALYSIS

\title{
SCHATTEN-CLASS GENERALIZED VOLTERRA COMPANION INTEGRAL OPERATORS
}

\author{
TESFA MENGESTIE
}

Communicated by R. E. Curto

\begin{abstract}
We study the Schatten-class membership of generalized Volterra companion integral operators on the standard Fock spaces $\mathcal{F}_{\alpha}^{2}$. The Schatten $\mathcal{S}_{p}\left(\mathcal{F}_{\alpha}^{2}\right)$ membership of the operators are characterized in terms of $L^{p / 2}$-integrability of certain generalized Berezin-type integral transforms on the complex plane. We also give a more simplified and easy-to-apply description in terms of $L^{p}$-integrability of the symbols inducing the operators against super-exponentially decreasing weights. Asymptotic estimates for the $\mathcal{S}_{p}\left(\mathcal{F}_{\alpha}^{2}\right)$ norms of the operators have also been provided.
\end{abstract}

\section{INTRODUCTION AND MAIN RESULTS}

For functions $f$ and $g$, we consider the Volterra-type integral operator $V_{g}$ and its companion $I_{g}$ defined by

$$
V_{g} f(z)=\int_{0}^{z} f(w) g^{\prime}(w) d w \quad \text { and } \quad I_{g} f(z)=\int_{0}^{z} f^{\prime}(w) g(w) d w .
$$

Performing integration by parts in any one of the above integrals gives the relation

$$
V_{g} f+I_{g} f=M_{g} f-f(0) g(0)
$$

where $M_{g} f=g f$ is the multiplication operator induced by $g$. These integral operators have been studied extensively on various spaces of holomorphic functions with the aim to explore the connection between their behaviors with the functiontheoretic properties of the symbols $g$, especially after the works of Pommerenke

Copyright 2016 by the Tusi Mathematical Research Group.

Received Apr. 11, 2015; Accepted Jun. 7, 2015.

2010 Mathematics Subject Classification. Primary 47B32; Secondary 46E22, 46E20, 47B33.

Keywords. Fock space, Schatten class, Berezin transform, Volterra operator, generalized Volterra companion operator. 


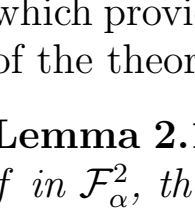

MATHEMATICAL

ANALYSIS

\title{
SCHATTEN-CLASS GENERALIZED VOLTERRA COMPANION INTEGRAL OPERATORS
}

\author{
TESFA MENGESTIE
}

Communicated by R. E. Curto

\begin{abstract}
We study the Schatten-class membership of generalized Volterra companion integral operators on the standard Fock spaces $\mathcal{F}_{\alpha}^{2}$. The Schatten $\mathcal{S}_{p}\left(\mathcal{F}_{\alpha}^{2}\right)$ membership of the operators are characterized in terms of $L^{p / 2}$-integrability of certain generalized Berezin-type integral transforms on the complex plane. We also give a more simplified and easy-to-apply description in terms of $L^{p}$-integrability of the symbols inducing the operators against super-exponentially decreasing weights. Asymptotic estimates for the $\mathcal{S}_{p}\left(\mathcal{F}_{\alpha}^{2}\right)$ norms of the operators have also been provided.
\end{abstract}

\section{INTRODUCTION AND MAIN RESULTS}

For functions $f$ and $g$, we consider the Volterra-type integral operator $V_{g}$ and its companion $I_{g}$ defined by

$$
V_{g} f(z)=\int_{0}^{z} f(w) g^{\prime}(w) d w \quad \text { and } \quad I_{g} f(z)=\int_{0}^{z} f^{\prime}(w) g(w) d w .
$$

Performing integration by parts in any one of the above integrals gives the relation

$$
V_{g} f+I_{g} f=M_{g} f-f(0) g(0)
$$

where $M_{g} f=g f$ is the multiplication operator induced by $g$. These integral operators have been studied extensively on various spaces of holomorphic functions with the aim to explore the connection between their behaviors with the functiontheoretic properties of the symbols $g$, especially after the works of Pommerenke

Copyright 2016 by the Tusi Mathematical Research Group.

Received Apr. 11, 2015; Accepted Jun. 7, 2015.

2010 Mathematics Subject Classification. Primary 47B32; Secondary 46E22, 46E20, 47B33.

Keywords. Fock space, Schatten class, Berezin transform, Volterra operator, generalized Volterra companion operator. 


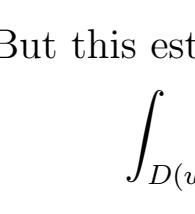

MATHEMATICAL

ANALYSIS

\title{
SCHATTEN-CLASS GENERALIZED VOLTERRA COMPANION INTEGRAL OPERATORS
}

\author{
TESFA MENGESTIE
}

Communicated by R. E. Curto

\begin{abstract}
We study the Schatten-class membership of generalized Volterra companion integral operators on the standard Fock spaces $\mathcal{F}_{\alpha}^{2}$. The Schatten $\mathcal{S}_{p}\left(\mathcal{F}_{\alpha}^{2}\right)$ membership of the operators are characterized in terms of $L^{p / 2}$-integrability of certain generalized Berezin-type integral transforms on the complex plane. We also give a more simplified and easy-to-apply description in terms of $L^{p}$-integrability of the symbols inducing the operators against super-exponentially decreasing weights. Asymptotic estimates for the $\mathcal{S}_{p}\left(\mathcal{F}_{\alpha}^{2}\right)$ norms of the operators have also been provided.
\end{abstract}

\section{INTRODUCTION AND MAIN RESULTS}

For functions $f$ and $g$, we consider the Volterra-type integral operator $V_{g}$ and its companion $I_{g}$ defined by

$$
V_{g} f(z)=\int_{0}^{z} f(w) g^{\prime}(w) d w \quad \text { and } \quad I_{g} f(z)=\int_{0}^{z} f^{\prime}(w) g(w) d w .
$$

Performing integration by parts in any one of the above integrals gives the relation

$$
V_{g} f+I_{g} f=M_{g} f-f(0) g(0)
$$

where $M_{g} f=g f$ is the multiplication operator induced by $g$. These integral operators have been studied extensively on various spaces of holomorphic functions with the aim to explore the connection between their behaviors with the functiontheoretic properties of the symbols $g$, especially after the works of Pommerenke

Copyright 2016 by the Tusi Mathematical Research Group.

Received Apr. 11, 2015; Accepted Jun. 7, 2015.

2010 Mathematics Subject Classification. Primary 47B32; Secondary 46E22, 46E20, 47B33.

Keywords. Fock space, Schatten class, Berezin transform, Volterra operator, generalized Volterra companion operator. 


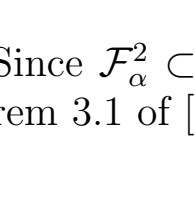

MATHEMATICAL

ANALYSIS

\title{
SCHATTEN-CLASS GENERALIZED VOLTERRA COMPANION INTEGRAL OPERATORS
}

\author{
TESFA MENGESTIE
}

Communicated by R. E. Curto

\begin{abstract}
We study the Schatten-class membership of generalized Volterra companion integral operators on the standard Fock spaces $\mathcal{F}_{\alpha}^{2}$. The Schatten $\mathcal{S}_{p}\left(\mathcal{F}_{\alpha}^{2}\right)$ membership of the operators are characterized in terms of $L^{p / 2}$-integrability of certain generalized Berezin-type integral transforms on the complex plane. We also give a more simplified and easy-to-apply description in terms of $L^{p}$-integrability of the symbols inducing the operators against super-exponentially decreasing weights. Asymptotic estimates for the $\mathcal{S}_{p}\left(\mathcal{F}_{\alpha}^{2}\right)$ norms of the operators have also been provided.
\end{abstract}

\section{INTRODUCTION AND MAIN RESULTS}

For functions $f$ and $g$, we consider the Volterra-type integral operator $V_{g}$ and its companion $I_{g}$ defined by

$$
V_{g} f(z)=\int_{0}^{z} f(w) g^{\prime}(w) d w \quad \text { and } \quad I_{g} f(z)=\int_{0}^{z} f^{\prime}(w) g(w) d w .
$$

Performing integration by parts in any one of the above integrals gives the relation

$$
V_{g} f+I_{g} f=M_{g} f-f(0) g(0)
$$

where $M_{g} f=g f$ is the multiplication operator induced by $g$. These integral operators have been studied extensively on various spaces of holomorphic functions with the aim to explore the connection between their behaviors with the functiontheoretic properties of the symbols $g$, especially after the works of Pommerenke

Copyright 2016 by the Tusi Mathematical Research Group.

Received Apr. 11, 2015; Accepted Jun. 7, 2015.

2010 Mathematics Subject Classification. Primary 47B32; Secondary 46E22, 46E20, 47B33.

Keywords. Fock space, Schatten class, Berezin transform, Volterra operator, generalized Volterra companion operator. 


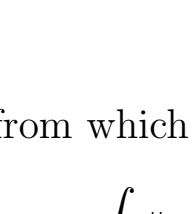

MATHEMATICAL

ANALYSIS

\title{
SCHATTEN-CLASS GENERALIZED VOLTERRA COMPANION INTEGRAL OPERATORS
}

\author{
TESFA MENGESTIE
}

Communicated by R. E. Curto

\begin{abstract}
We study the Schatten-class membership of generalized Volterra companion integral operators on the standard Fock spaces $\mathcal{F}_{\alpha}^{2}$. The Schatten $\mathcal{S}_{p}\left(\mathcal{F}_{\alpha}^{2}\right)$ membership of the operators are characterized in terms of $L^{p / 2}$-integrability of certain generalized Berezin-type integral transforms on the complex plane. We also give a more simplified and easy-to-apply description in terms of $L^{p}$-integrability of the symbols inducing the operators against super-exponentially decreasing weights. Asymptotic estimates for the $\mathcal{S}_{p}\left(\mathcal{F}_{\alpha}^{2}\right)$ norms of the operators have also been provided.
\end{abstract}

\section{INTRODUCTION AND MAIN RESULTS}

For functions $f$ and $g$, we consider the Volterra-type integral operator $V_{g}$ and its companion $I_{g}$ defined by

$$
V_{g} f(z)=\int_{0}^{z} f(w) g^{\prime}(w) d w \quad \text { and } \quad I_{g} f(z)=\int_{0}^{z} f^{\prime}(w) g(w) d w .
$$

Performing integration by parts in any one of the above integrals gives the relation

$$
V_{g} f+I_{g} f=M_{g} f-f(0) g(0)
$$

where $M_{g} f=g f$ is the multiplication operator induced by $g$. These integral operators have been studied extensively on various spaces of holomorphic functions with the aim to explore the connection between their behaviors with the functiontheoretic properties of the symbols $g$, especially after the works of Pommerenke

Copyright 2016 by the Tusi Mathematical Research Group.

Received Apr. 11, 2015; Accepted Jun. 7, 2015.

2010 Mathematics Subject Classification. Primary 47B32; Secondary 46E22, 46E20, 47B33.

Keywords. Fock space, Schatten class, Berezin transform, Volterra operator, generalized Volterra companion operator. 


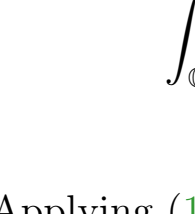

MATHEMATICAL

ANALYSIS

\title{
SCHATTEN-CLASS GENERALIZED VOLTERRA COMPANION INTEGRAL OPERATORS
}

\author{
TESFA MENGESTIE
}

Communicated by R. E. Curto

\begin{abstract}
We study the Schatten-class membership of generalized Volterra companion integral operators on the standard Fock spaces $\mathcal{F}_{\alpha}^{2}$. The Schatten $\mathcal{S}_{p}\left(\mathcal{F}_{\alpha}^{2}\right)$ membership of the operators are characterized in terms of $L^{p / 2}$-integrability of certain generalized Berezin-type integral transforms on the complex plane. We also give a more simplified and easy-to-apply description in terms of $L^{p}$-integrability of the symbols inducing the operators against super-exponentially decreasing weights. Asymptotic estimates for the $\mathcal{S}_{p}\left(\mathcal{F}_{\alpha}^{2}\right)$ norms of the operators have also been provided.
\end{abstract}

\section{INTRODUCTION AND MAIN RESULTS}

For functions $f$ and $g$, we consider the Volterra-type integral operator $V_{g}$ and its companion $I_{g}$ defined by

$$
V_{g} f(z)=\int_{0}^{z} f(w) g^{\prime}(w) d w \quad \text { and } \quad I_{g} f(z)=\int_{0}^{z} f^{\prime}(w) g(w) d w .
$$

Performing integration by parts in any one of the above integrals gives the relation

$$
V_{g} f+I_{g} f=M_{g} f-f(0) g(0)
$$

where $M_{g} f=g f$ is the multiplication operator induced by $g$. These integral operators have been studied extensively on various spaces of holomorphic functions with the aim to explore the connection between their behaviors with the functiontheoretic properties of the symbols $g$, especially after the works of Pommerenke

Copyright 2016 by the Tusi Mathematical Research Group.

Received Apr. 11, 2015; Accepted Jun. 7, 2015.

2010 Mathematics Subject Classification. Primary 47B32; Secondary 46E22, 46E20, 47B33.

Keywords. Fock space, Schatten class, Berezin transform, Volterra operator, generalized Volterra companion operator. 


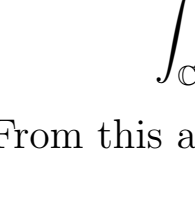

MATHEMATICAL

ANALYSIS

\title{
SCHATTEN-CLASS GENERALIZED VOLTERRA COMPANION INTEGRAL OPERATORS
}

\author{
TESFA MENGESTIE
}

Communicated by R. E. Curto

\begin{abstract}
We study the Schatten-class membership of generalized Volterra companion integral operators on the standard Fock spaces $\mathcal{F}_{\alpha}^{2}$. The Schatten $\mathcal{S}_{p}\left(\mathcal{F}_{\alpha}^{2}\right)$ membership of the operators are characterized in terms of $L^{p / 2}$-integrability of certain generalized Berezin-type integral transforms on the complex plane. We also give a more simplified and easy-to-apply description in terms of $L^{p}$-integrability of the symbols inducing the operators against super-exponentially decreasing weights. Asymptotic estimates for the $\mathcal{S}_{p}\left(\mathcal{F}_{\alpha}^{2}\right)$ norms of the operators have also been provided.
\end{abstract}

\section{INTRODUCTION AND MAIN RESULTS}

For functions $f$ and $g$, we consider the Volterra-type integral operator $V_{g}$ and its companion $I_{g}$ defined by

$$
V_{g} f(z)=\int_{0}^{z} f(w) g^{\prime}(w) d w \quad \text { and } \quad I_{g} f(z)=\int_{0}^{z} f^{\prime}(w) g(w) d w .
$$

Performing integration by parts in any one of the above integrals gives the relation

$$
V_{g} f+I_{g} f=M_{g} f-f(0) g(0)
$$

where $M_{g} f=g f$ is the multiplication operator induced by $g$. These integral operators have been studied extensively on various spaces of holomorphic functions with the aim to explore the connection between their behaviors with the functiontheoretic properties of the symbols $g$, especially after the works of Pommerenke

Copyright 2016 by the Tusi Mathematical Research Group.

Received Apr. 11, 2015; Accepted Jun. 7, 2015.

2010 Mathematics Subject Classification. Primary 47B32; Secondary 46E22, 46E20, 47B33.

Keywords. Fock space, Schatten class, Berezin transform, Volterra operator, generalized Volterra companion operator. 


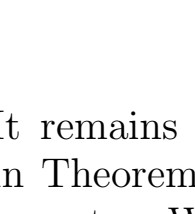

MATHEMATICAL

ANALYSIS

\title{
SCHATTEN-CLASS GENERALIZED VOLTERRA COMPANION INTEGRAL OPERATORS
}

\author{
TESFA MENGESTIE
}

Communicated by R. E. Curto

\begin{abstract}
We study the Schatten-class membership of generalized Volterra companion integral operators on the standard Fock spaces $\mathcal{F}_{\alpha}^{2}$. The Schatten $\mathcal{S}_{p}\left(\mathcal{F}_{\alpha}^{2}\right)$ membership of the operators are characterized in terms of $L^{p / 2}$-integrability of certain generalized Berezin-type integral transforms on the complex plane. We also give a more simplified and easy-to-apply description in terms of $L^{p}$-integrability of the symbols inducing the operators against super-exponentially decreasing weights. Asymptotic estimates for the $\mathcal{S}_{p}\left(\mathcal{F}_{\alpha}^{2}\right)$ norms of the operators have also been provided.
\end{abstract}

\section{INTRODUCTION AND MAIN RESULTS}

For functions $f$ and $g$, we consider the Volterra-type integral operator $V_{g}$ and its companion $I_{g}$ defined by

$$
V_{g} f(z)=\int_{0}^{z} f(w) g^{\prime}(w) d w \quad \text { and } \quad I_{g} f(z)=\int_{0}^{z} f^{\prime}(w) g(w) d w .
$$

Performing integration by parts in any one of the above integrals gives the relation

$$
V_{g} f+I_{g} f=M_{g} f-f(0) g(0)
$$

where $M_{g} f=g f$ is the multiplication operator induced by $g$. These integral operators have been studied extensively on various spaces of holomorphic functions with the aim to explore the connection between their behaviors with the functiontheoretic properties of the symbols $g$, especially after the works of Pommerenke

Copyright 2016 by the Tusi Mathematical Research Group.

Received Apr. 11, 2015; Accepted Jun. 7, 2015.

2010 Mathematics Subject Classification. Primary 47B32; Secondary 46E22, 46E20, 47B33.

Keywords. Fock space, Schatten class, Berezin transform, Volterra operator, generalized Volterra companion operator. 


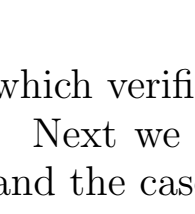

MATHEMATICAL

ANALYSIS

\title{
SCHATTEN-CLASS GENERALIZED VOLTERRA COMPANION INTEGRAL OPERATORS
}

\author{
TESFA MENGESTIE
}

Communicated by R. E. Curto

\begin{abstract}
We study the Schatten-class membership of generalized Volterra companion integral operators on the standard Fock spaces $\mathcal{F}_{\alpha}^{2}$. The Schatten $\mathcal{S}_{p}\left(\mathcal{F}_{\alpha}^{2}\right)$ membership of the operators are characterized in terms of $L^{p / 2}$-integrability of certain generalized Berezin-type integral transforms on the complex plane. We also give a more simplified and easy-to-apply description in terms of $L^{p}$-integrability of the symbols inducing the operators against super-exponentially decreasing weights. Asymptotic estimates for the $\mathcal{S}_{p}\left(\mathcal{F}_{\alpha}^{2}\right)$ norms of the operators have also been provided.
\end{abstract}

\section{INTRODUCTION AND MAIN RESULTS}

For functions $f$ and $g$, we consider the Volterra-type integral operator $V_{g}$ and its companion $I_{g}$ defined by

$$
V_{g} f(z)=\int_{0}^{z} f(w) g^{\prime}(w) d w \quad \text { and } \quad I_{g} f(z)=\int_{0}^{z} f^{\prime}(w) g(w) d w .
$$

Performing integration by parts in any one of the above integrals gives the relation

$$
V_{g} f+I_{g} f=M_{g} f-f(0) g(0)
$$

where $M_{g} f=g f$ is the multiplication operator induced by $g$. These integral operators have been studied extensively on various spaces of holomorphic functions with the aim to explore the connection between their behaviors with the functiontheoretic properties of the symbols $g$, especially after the works of Pommerenke

Copyright 2016 by the Tusi Mathematical Research Group.

Received Apr. 11, 2015; Accepted Jun. 7, 2015.

2010 Mathematics Subject Classification. Primary 47B32; Secondary 46E22, 46E20, 47B33.

Keywords. Fock space, Schatten class, Berezin transform, Volterra operator, generalized Volterra companion operator. 


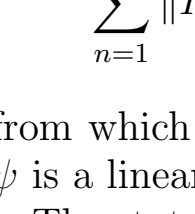

MATHEMATICAL

ANALYSIS

\title{
SCHATTEN-CLASS GENERALIZED VOLTERRA COMPANION INTEGRAL OPERATORS
}

\author{
TESFA MENGESTIE
}

Communicated by R. E. Curto

\begin{abstract}
We study the Schatten-class membership of generalized Volterra companion integral operators on the standard Fock spaces $\mathcal{F}_{\alpha}^{2}$. The Schatten $\mathcal{S}_{p}\left(\mathcal{F}_{\alpha}^{2}\right)$ membership of the operators are characterized in terms of $L^{p / 2}$-integrability of certain generalized Berezin-type integral transforms on the complex plane. We also give a more simplified and easy-to-apply description in terms of $L^{p}$-integrability of the symbols inducing the operators against super-exponentially decreasing weights. Asymptotic estimates for the $\mathcal{S}_{p}\left(\mathcal{F}_{\alpha}^{2}\right)$ norms of the operators have also been provided.
\end{abstract}

\section{INTRODUCTION AND MAIN RESULTS}

For functions $f$ and $g$, we consider the Volterra-type integral operator $V_{g}$ and its companion $I_{g}$ defined by

$$
V_{g} f(z)=\int_{0}^{z} f(w) g^{\prime}(w) d w \quad \text { and } \quad I_{g} f(z)=\int_{0}^{z} f^{\prime}(w) g(w) d w .
$$

Performing integration by parts in any one of the above integrals gives the relation

$$
V_{g} f+I_{g} f=M_{g} f-f(0) g(0)
$$

where $M_{g} f=g f$ is the multiplication operator induced by $g$. These integral operators have been studied extensively on various spaces of holomorphic functions with the aim to explore the connection between their behaviors with the functiontheoretic properties of the symbols $g$, especially after the works of Pommerenke

Copyright 2016 by the Tusi Mathematical Research Group.

Received Apr. 11, 2015; Accepted Jun. 7, 2015.

2010 Mathematics Subject Classification. Primary 47B32; Secondary 46E22, 46E20, 47B33.

Keywords. Fock space, Schatten class, Berezin transform, Volterra operator, generalized Volterra companion operator. 


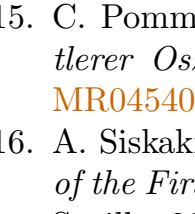

MATHEMATICAL

ANALYSIS

\title{
SCHATTEN-CLASS GENERALIZED VOLTERRA COMPANION INTEGRAL OPERATORS
}

\author{
TESFA MENGESTIE
}

Communicated by R. E. Curto

\begin{abstract}
We study the Schatten-class membership of generalized Volterra companion integral operators on the standard Fock spaces $\mathcal{F}_{\alpha}^{2}$. The Schatten $\mathcal{S}_{p}\left(\mathcal{F}_{\alpha}^{2}\right)$ membership of the operators are characterized in terms of $L^{p / 2}$-integrability of certain generalized Berezin-type integral transforms on the complex plane. We also give a more simplified and easy-to-apply description in terms of $L^{p}$-integrability of the symbols inducing the operators against super-exponentially decreasing weights. Asymptotic estimates for the $\mathcal{S}_{p}\left(\mathcal{F}_{\alpha}^{2}\right)$ norms of the operators have also been provided.
\end{abstract}

\section{INTRODUCTION AND MAIN RESULTS}

For functions $f$ and $g$, we consider the Volterra-type integral operator $V_{g}$ and its companion $I_{g}$ defined by

$$
V_{g} f(z)=\int_{0}^{z} f(w) g^{\prime}(w) d w \quad \text { and } \quad I_{g} f(z)=\int_{0}^{z} f^{\prime}(w) g(w) d w .
$$

Performing integration by parts in any one of the above integrals gives the relation

$$
V_{g} f+I_{g} f=M_{g} f-f(0) g(0)
$$

where $M_{g} f=g f$ is the multiplication operator induced by $g$. These integral operators have been studied extensively on various spaces of holomorphic functions with the aim to explore the connection between their behaviors with the functiontheoretic properties of the symbols $g$, especially after the works of Pommerenke

Copyright 2016 by the Tusi Mathematical Research Group.

Received Apr. 11, 2015; Accepted Jun. 7, 2015.

2010 Mathematics Subject Classification. Primary 47B32; Secondary 46E22, 46E20, 47B33.

Keywords. Fock space, Schatten class, Berezin transform, Volterra operator, generalized Volterra companion operator. 